\title{
Fruit Fly Identification from Fruits and Vegetables of Turikale Maros, South Sulawesi, Indonesia
}

\author{
Itji Diana Daud ${ }^{1 *}$, Melina $^{1}$, Dayanara Haditama Khomsah ${ }^{1}$, Mustika Tuwo $^{2}$ \\ ${ }^{1}$ Department of Plants Pests and Diseases, Faculty of Agriculture, Hasanuddin University, Makassar, South \\ Sulawesi 90245, Indonesia \\ ${ }^{2}$ Department of Biology, Faculty of Mathematics and Natural Sciences, Hasanuddin University, Makassar, South \\ Sulawesi 90245, Indonesia \\ *Corresponding author.Email: itfir@yahoo.com
}

\begin{abstract}
Indonesia has an abundant production of fruits and vegetables that can satisfy the demands of domestic and export markets. One of the most serious problems in fruit exports is the damage and contamination caused by fruit fly attacks to horticultural produce. Taiwan government rejects 13 types of fruit and vegetable commodities from Indonesia due to infestation by several species of fruit fly, including Bactrocera papayae, B. zonata, and B. musae. The research was carried out in Turikale, Maros District, South Sulawesi, Indonesia. Various fruits and vegetables serving as hosts of fruit flies, including Capsicum annum (chilli), Solanum lycopersium (tomato), Avverhoa carambola (star fruit), Psidium guajava (guava), Artocarpus heterophylllus (jackfruit), are cultivated in the area. The study was initiated to identify the fruit fly species associated with vegetable and fruit crops in the study site. Fruits and vegetables attacked by fruit flies were collected from the field and then separately kept in plastic containers in the laboratory until the adults emerged. The emerging flies were identified under a dissecting microscope based on the identification keys. Five species of fruit flies belonging to the Genus Bactorcera emerged from the collected fruits and vegetables, namely: B. albistrigata emerging from Psidium guajava and Syzygium aqueum; B. dorsalis emerging from Avverhoa carambola, Mangifera indica, Capsicum annum, and Solanum melongena; B. cucurbitae emerging from Momordica charatina; B. carambolae emerging from Artocarpus heterophyllus; and B. umbrosa emerging from Artocarpus altilis.
\end{abstract}

Keywords: Bactrocera, holometabola, morphology, Mangifera indica, Capsicum annum

\section{INTRODUCTION}

Indonesia, as a tropical country, has a variety of fruits and vegetables. Efforts to increase production both to meet domestic needs and for exports constantly face with serious obstacles, for instance, the attack of various species of fruit flies reduces the fruit and vegetable products both qualitatively and quantitatively. Fruits or vegetables infested by fruit flies is initially damaged by the larval activity but the damage is then intensified by the activity of decaying microorganisms associated with the fruit fly. Thus, fruits and vegetables attacked by the fruit fly lose their economic and aesthetic values.

There have been many cases of export rejection of fresh fruits and vegetables by an importing country due to the presence of pests in the products, including the fruit flies [1]. Thirteen Indonesian agricultural commodities are threatened to be rejected by the Taiwanese Government after nine species of fruit fly were detected in the products. Indonesia is suspected of having three species of fruit flies that do not yet exist in Taiwan; these species are $B$. papayae, B. zonata, and B. musae. Fruit that is attacked by fruit flies also has an impact on export destination countries such as China, Japan, Korea, and Taiwan. Fruit flies are one of the pests that are very difficult to control, even in Japan, Australia, and New Zealand, the governments have spent substantial amount of funds to cope with the damage caused by the pest [2]. There are 77 species of fruit flies of the genus Bactrocera spread throughout Indonesia, such as Sumatra, Java, Kalimantan, Sulawesi, Maluku, Nusa Tenggara, and Irian which attack various kinds of fruits and vegetables [3]. In Tomohon City, North Suawesi Province, 10 species of Bactrocera were found on chili, tomato, and squash [4].

According to Khaerudin et al. [5], B. dorsalis, B. carambolae, B. albistrigata, $B$. cucurbitae, and $B$. emittens (polyphagous), and B. latifrons (oligophagous) were found in West Sulawesi Province. Among those species, B. dorsalis, B. carambolae, B. umbrosa, B. albistrigata, and B. limbifera are the dominant species. A great deal of research has been conducted to assess and identify fruit flies species and damage to various fruits and vegetables throughout Indonesia. However, in South Sulawesi assessments have been carried out in a few areas such as Enrekang, Soppeng, and Wajo districts. Sri [6] found $B$. dorsalis on mango, star-fruits, cashews, cayenne 
peppers, and red chilies; $B$. albistrigata in guavas; $B$. umbrosa on breadfruits and jackfruits; and $D$. melanogaster on mangoes, tomatoes, and jackfruits in Soppeng District. Whereas in Wajo District, $B$. dorsalis was found on cayenne peppers, mangoes and chilies; $B$. papaya on chilies; $B$. umbrosa on jackfruits and breadfruits; $B$. albistrigata in guavas; and $D$. melanogaster on mangoes, papayas, jackfruits, star-fruits, and tomatoes [7].

Based on research conducted by Yuniar [8] in Enrekang District, B. carambolae was found in avocados and papayas; B.dorsalis on mango, starfruit, cayenne pepper, red chili, guava, tomato, and grapefruit; $B$. umbrosa on jackfruit and red chili; B. albistrigata in guava; and $D$. melanogaster in tomatoes and water guava.

Maros District, especially Turikale Sub-district, has quite a high diversity of fruit and vegetable plants such as chili, tomatoes, starfruit, guava, white guava, jackfruit, and other crops that are suitable for the development of fruit flies. This study was conducted to identify the species of the fruit flies infesting fruits and vegetables in Maros district. The results of the study can be used as a basis for constructing an anticipatory action of producing high quality fruit and vegetable products that are competitive in domestic and international markets.

\subsection{Materials and Methods}

The study was conducted during fruiting season (AprilSeptember 2017) on Turikale Maros, South Sulawesi. A large infested fruit and vegetable samples were collected such as Capsicum annum, Solanum melongena, and Momordica charatina were gathered from gardens, while Psidium guajava, Syzygium aqueum, Avverhoa carambola, Mangifera indica, Artocarpus altilis, and Artocarpus heterophyllus were acquired from the residential yards. Each sample was placed in different plastic bags. Each collected fruits and vegetables were transferred to a plastic container that contains $3-5 \mathrm{~cm}$ of filtered sterile sand. The top part of the plastic container was covered with screen. After $12 \mathrm{~d}$ of incubation, we observed the plastic container every day until adult emerged (Figure 1).

Newly emerged adult fed with honey in order to get a good color pattern on the thorax and abdomen. Cotton

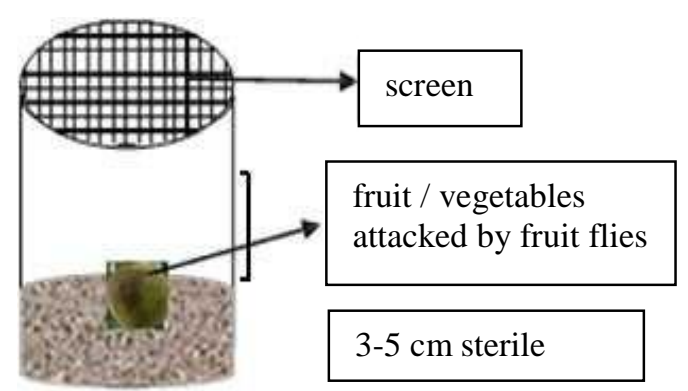

Figure 1 Plastic containers were containing sterile sand $3-5 \mathrm{~cm}$ high soaked in ethanol were put into containers to knock the fruit flies out. The dead fruit flies were identified under a microscope using the identification key of fruit flies [9]. Once the determination completed, the flies were pinned and stored in a collection box.

\subsection{Our Contribution}

This paper served the method to collect fruit flies from infested fruits and vegetables. This paper also showed photographic documentation along with the description of the main characteristics of identified fruit flies species which emerged from their host plants.

\subsection{Paper Structure}

The rest of the paper is organized as follows: section 2 presents identification results and descriptions of fruit flies species i.e. B. albistrigata, B. dorsalis, B. cucurbitae, B. umbrosa, and $B$. carambolae. The description is provided with photographic documentation of key characteristics of the species: section 3 concludes the paper and presents direction for future research.

\section{RESULTS AND DISCUSSION}

The identification results show that in Turikale Subdistrict, Maros District, five species of fruit flies were found, i.e., Bactrocera. albistrigata, B. carambolae, B. cucurbitae, B. dorsalis, and B. umbrosa, which belong to genus Bactrocera and two subgenera Bactrocera and Zeugodacus (Table 1). All of the species were found attacking different hosts. Bactrocera dorsalis is an oriental fruit fly with wide range of hosts. In Turikale Sub-district, Maros District, $B$. dorsalis were found attacking starfruit, mango, large chili, and eggplant. This result is in line with the statement of Khaeruddin et al. [5], which states that $B$. dorsalis is polyphagous. It means $B$. dorsalis can live and breed on more than one species of host plant from one or several families. Meanwhile, $B$. albistrigata and $B$. carambolae are also known as polyphagous insects, but in this study, we only found them attacking only one host; Psidium guajava and Artocarpus heterophyllus, respectively. As for B. cucurbitae with host range only from family Cucurbitaceae and the hosts of $B$. umbrosa restricted to family Moraceae. These two species are known as oligophagous pests because they only attack several host species from the same family [10].

Overall morphological characteristics of each species are described as follows.

Bactrocera albistrigata. Head with a pair of compound eyes with aristate antennae, and a pair of oval-shaped black spots (Figure 2). Lateral postsutural vittae present and yellow; scutum blackish brown; no presutural vittae in notopleuron; scutellum is pale yellow with a black base (Figure 3 ). The abdomen is dominated by yellow-orange. 
In the III-V segment, there is a narrow dark medial line and medium width dark lateral bands (Figure 4).

The wing is mostly brownish. The costal band on R1 is pale but darker than the anal part. The number of transverse bands from the costal band to the underside of the wing consists of 2 bands, namely cubital streak and only. Transverse bands passing $\mathrm{r}-\mathrm{m}$ and $\mathrm{dm}-\mathrm{cu}$ at once (Figure 5).

Bactrocera dorsalis. The thorax of $B$. dorsalis, in the black-brown scutum, there is a pair of yellow postpronotal lobes, mesopleural stripe as wide as the middle distance between notopleuron and postpronotal lobe, there is a pair of lateral postsutular vittae (Figure 6). The abdomen is brown-orange; transverse black band between segments II and III; a long black medial longitudinal narrow dividing in the middle of segments III-V (Figure 7). Brownish transparent wings with black vessels. The costal width of the wing band does not reach $\mathrm{R} 4+5$; there is no wing pattern other than the costal band and cubital streak. It has a spot on the wingtips (Figure 8). According to [1], the species has black scutum, black mesonotum (middle thorax), yellow lateral band on the mesonotum extending near supra alar hair, two pairs of hairs on the inner frontoorbital, two hairs on the scutellum. The abdomen is mostly pale red (brown); there is a transverse black band on segment II and III; a longitudinal narrow black band dividing in the middle of segment III-V. The wings only have black bands on the lateral line and the anal line, do not have spots on the transverse veins.

Bactrocera (Zeugodacus) cucurbitae. Head has a pair of oval-shaped black spots, with aristate antennae and a pair of dichoptic compound eyes (Figure 9). Thorax has brown-red colored scutum with black-red markings appears. There are yellow bands on the lateral and medial postsutural vittae; the anterior end of the medial postsutural vittae are parallel to the base of the notopleuron seta, the scutellum is generally yellow (Figure 10). Abdomen segments III-V are brown-orange with a "T" shape consisting of a thin black line across the anterior margin of the third segment; the longitudinal medial line rather transversely wide in all three segments (Figure 11). The posterior tip of the side's wing pattern is rounded. Thin costal bands are blackish-brown; there is a fairly thick wing pattern on the $\mathrm{dm}-\mathrm{cu}$, there is also a wing pattern on the $r-m$ that looks very thin (Figure 12). Redbrown scutum. The lateral postsutural vittae narrows. There is a medial postsutural vittae. The costal band on the wing will grow paler between $\mathrm{R} 2+3$ and $\mathrm{R} 4+5$ which extends to meet a large spot on the wingtip. There is a dark band that extends from dm to cu. Cubital streak is wide and dark. The abdomen is orange-brown, there is a "T" pattern. Medial longitudinal dark band of medium size. The anterolateral angles in terga IV and V are dark in color. There is a pair of brightly coloured ceromae in the terga V. Dark abdominal sterna.

Bactrocera (Bactrocera) umbrosa Fabricius has a pair of small black oval-shaped spots. There are also aristate antennae and a pair of compound eyes (Figure 13). The scutum is black, the postpronotal lobe and notopleuron are yellow, the lateral postsuturalvittae are yellow with almost parallel width and stop just or slightly behind the intra alar seta, the mesopleural stripe is about to reach the postpronotal lobe, the yellow scutelum (Figure 14). Abdomen segments IV-V vary from orange to brown longitudinal median and a pair of shiny spot ceromae (shining spot) on segment V (Figure 15). Costal band that through R1 and R4+5, transverse band passing on middle of wings, transverse thin brown line in apex (Figure 16). The scutum is dominated by black, except in areas along the lateral and mesonotal sutures that have a brown colour. There is a lateral postsutural vittae that is wide and extends until it reaches or passes through the intra alar seta. The scutellum is yellow. The costal band on the wing is parallel to $\mathrm{R} 4+5$, accompanied by 3 additional bands that run from the costal wing to the underside of the wing. The abdomen is brown-orange and there is a medial longitudinal dark band that stretches from terga III to terga V. In terga $\mathrm{V}$, there is a pair of bright brown-orange ceromae.

Bactroera (Bactrocera) carambolae has a pair of small black oval-shaped spots. There are a pair of aristate antennae and a pair of separate compound eyes (figure 17). The thorax on the scutum is pale black with the back of the yellow ribbon; there is a pair of yellow postpronotal lobes and notopleuron, lateral postsutural vittae and postpronotal lobes are brown from the inside, yellow scutellum is yellow (Figure 18). The abdominal segments III-V are brown-orange and have a longitudinal medial and black anterior margin forming a " $\mathrm{T}$ " pattern, with transverse black bands in segments I and II (Figure 19). Brownish clear wings with brownish-black vessels slightly past $\mathrm{R} 2+3$ and slightly wider at the apex of R2 + 3 , which also passes through the apices of $\mathrm{R} 4+5$. There are no spots on the wingtips (Figure 20). The scutum is dominated by black and brown on the lateral back of the postsutural vittae, around the mesonotal suture and in the postpronotal lobes. There are lateral postsutural vittae with a type of parallel to the sub-parallel size of a medium to wide. Costal band on the wing overlaps with $\mathrm{R} 2+3$. There is a " $\mathrm{T}$ " pattern on the abdomen with a medial longitudinal dark band widening. The antero lateral angle in terga to IV is square. There is a pair of brightly coloured ceromae

Table 1 Fruit fly species attacking fruit and vegetable samples

\begin{tabular}{|l|l|}
\hline \multicolumn{1}{|c|}{ Species } & \multicolumn{1}{c|}{ Host plants } \\
\hline Bactrocera (Bactrocera) albistrigata & Psidium guajava, Syzygium aqueum \\
\hline Bactrocera (Bactrocera) dorsalis & Avverhoa carambola, Mangifera indica, Capsicum annum, Solanum melongena \\
\hline Bactrocera (Zeugodacus) cucurbitae & Momordica charantina \\
\hline Bactrocera (Bactrocera) umbrosa & Artocarpus altilis \\
\hline Bactrocera (Bactrocera) carambolae & Artocarpus heterophyllus \\
\hline
\end{tabular}




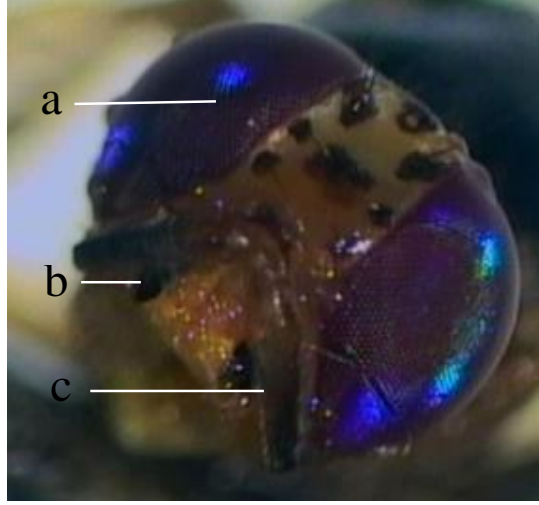

Figure 2 The anterior view of B. albistrigata head: (a) compound eyes, (b) a pair of spots, (c) aristate antennae

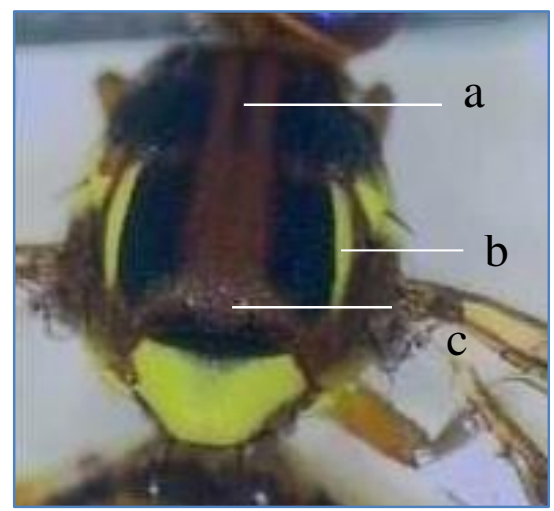

Figure 3 The dorsal view of B.albistrigata thorax: (a) scutum, (b) lateral postsutural vittae, (c) skutelum

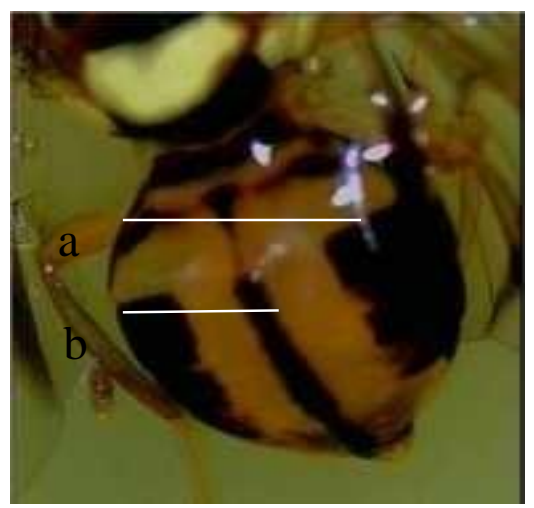

Figure 4 The dorsal view of $B$. albistrigata abdomen III-V: (a) medium width dark lateral bands, (b) a narrow dark medial line

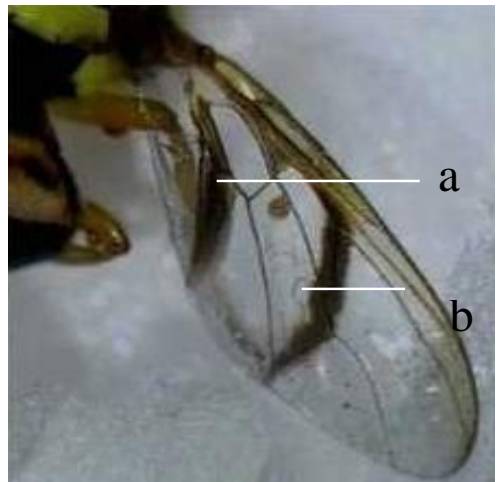

Figure 5 The wing of $B$. albistrigata: (a) broad anal transverse band, (b) transverse band passing $\mathrm{r}$ $\mathrm{m}$ and dm-cu

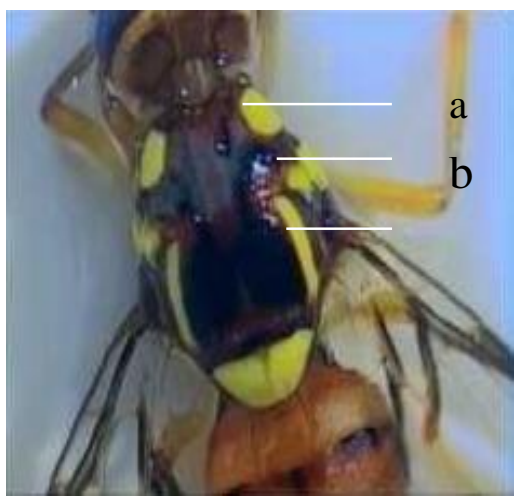

Figure 6 The dorsal view of $B$. dorsalis thorax: (a) yellow post-pronotallobe, (b) mesopleural stripe as wide as the middle distance between notopleuron and post-pronotal lobe

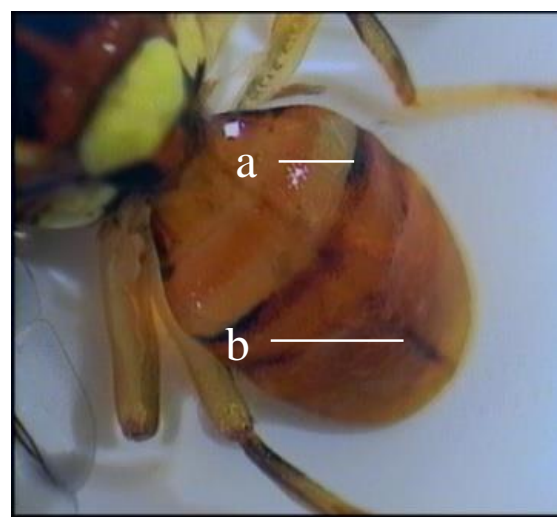

Figure 7 The dorsal view of $B$. dorsalis abdomen: (a) cross black band between segments II and III, (b) arrow longitudinal medial in segments III-V 


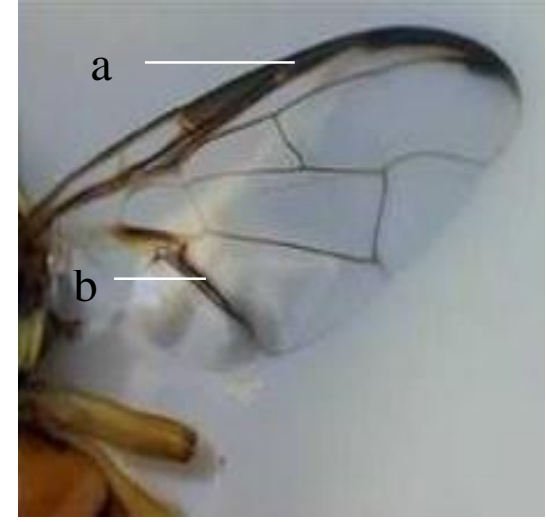

Figure 8 B. dorsalis wings: (a) costal band that passes through R1 and R2+3, (b) cubitalstreak

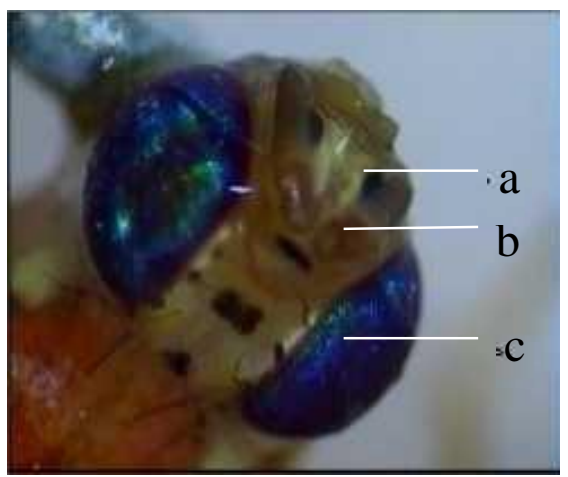

Figure 9 Frontal view of $B$. cucurbitae : (a) a pair of oval-shaped spots, (b) an aristate type antenna, (c) pair of dichoptic compound eyes

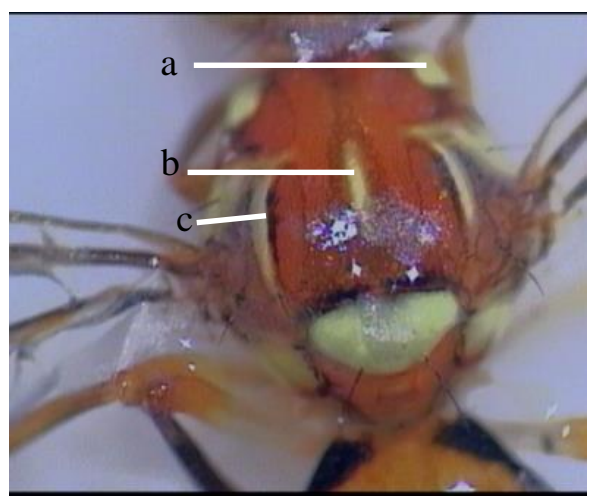

Figure 10 Dorsal view of B. cucurbitae thorax: (a) pair of yellow postpronotal lobes, (b) there is a medial postsuturalvittae, (c) a pair of lateral postsuturalvittae yellow with a brown-black interior

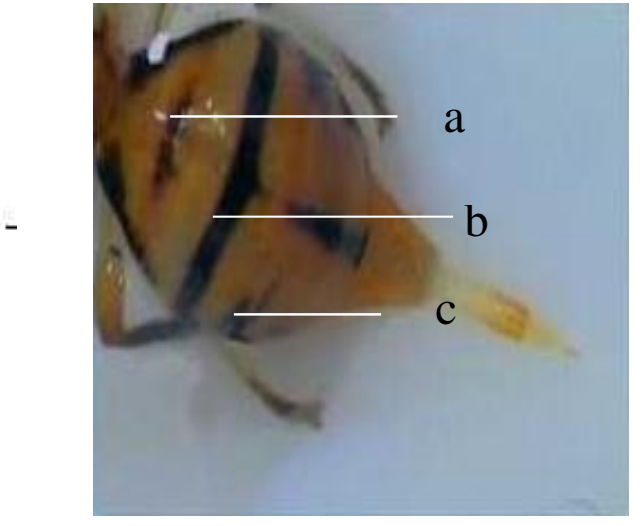

Figure 11 The dorsal view of $B$. cucurbitae abdomen: (a) longitudinal medial line, (b) anterior margin with pin III, (c) pair of anterolateral corners

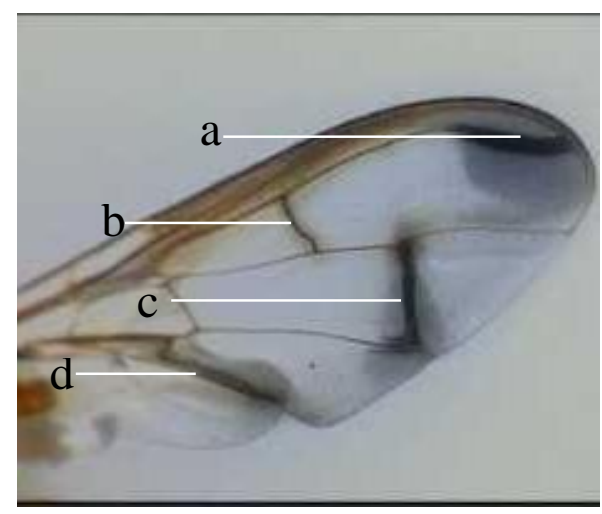

Figure 12 B. cucurbitae wings: (a) spot on wing tips, (b) wing pattern on rm, (c) additional bands on dm-cu, (d) cubitalstreak

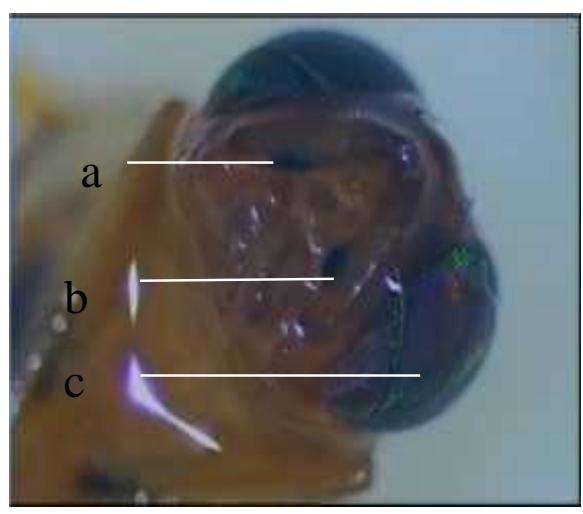

Figure 13 Frontal view of B. umbrosa head: (a) a pair of oval-shaped black spots, (b) aristate antennae, (c) separate compound eyes 


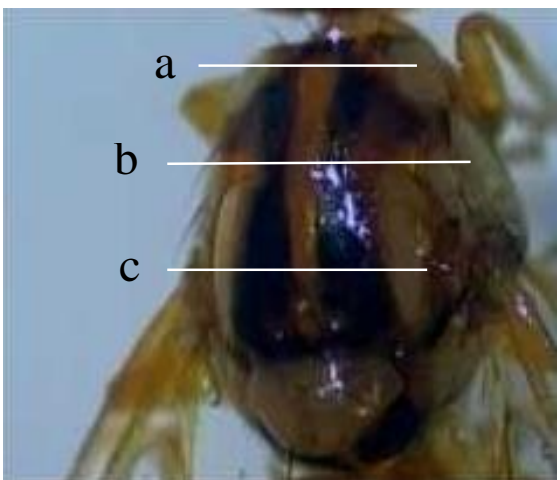

Figure 14 Dorsal view of $B$. umbrosa thorax: (a) post pronotal lobe in yellow, (b) mesopleural stripe with width almost reaching postpronotal lobe, (c) lateral postsutural vittae

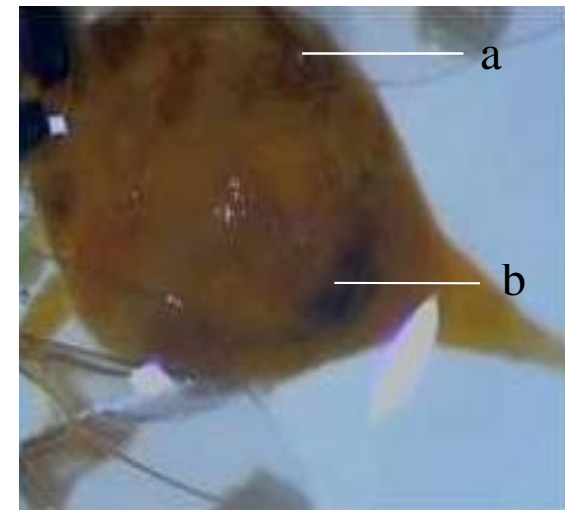

Figure 15 Dorsal view of $B$. umbrosa abdomen: (a) segment IV-V orange to brown longitudinal median, (b) ceromae on segment $\mathrm{V}$

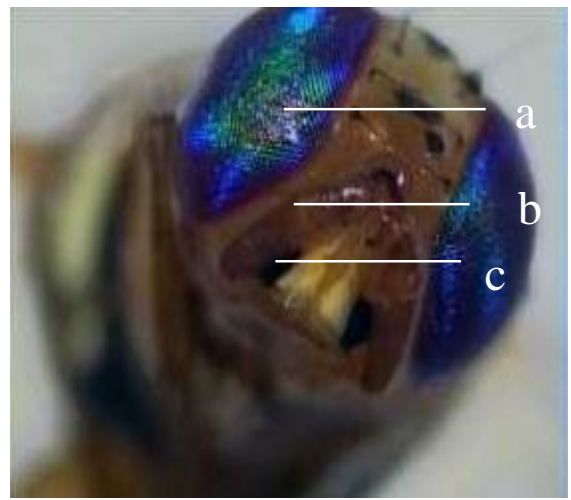

Figure 16 Frontal view of $B$. carambolae: (a) a pair of compound eyes, (b) an aristate antennae, (c) a pair of oval-shaped black spots

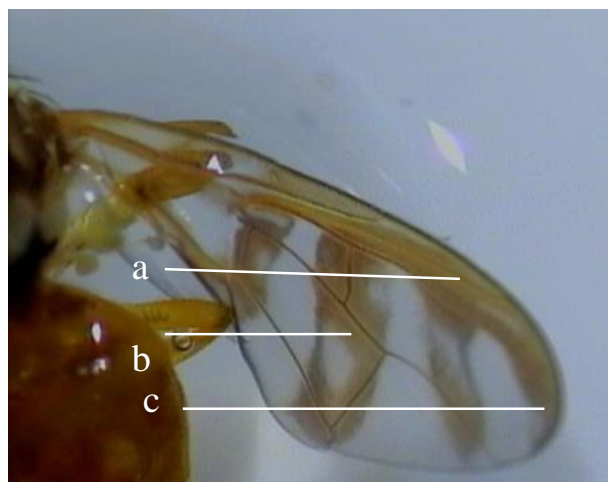

Figure 17 B. umbrosa wings: (a) costal band that through R1 and R4+5, (b) transverse band passing on middle of wings, (c) transverse thin brown line in apeks

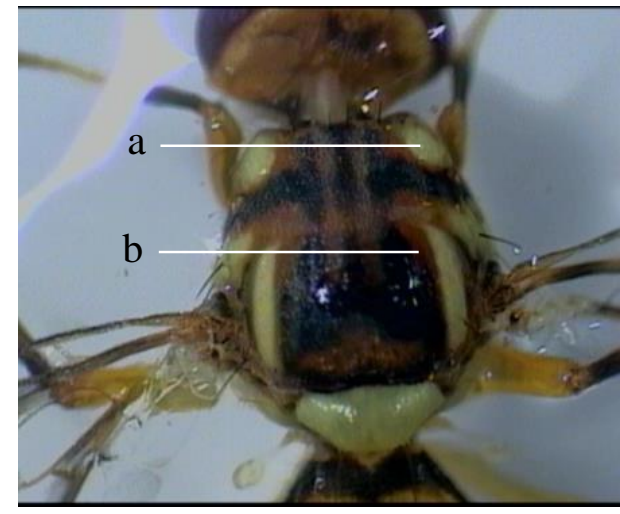

Figure 18 Thorax B. carambolae: (a) post pronotal lobe and notopleuron yellow, (b) the lateral side of the postsutural vittae and post pronotal lobe are brown from the inside

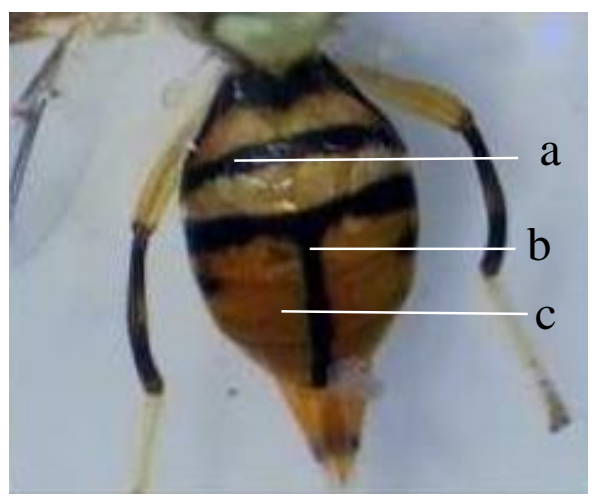

Figure 19 Dorsal view of $B$. carambolae abdomen: (a) black transverse band between segments I and II, (b) black anterior margins connected with medial longitudinal form the pattern of the letter " $\mathrm{T}$ ", (c) medial line from segments III-V 


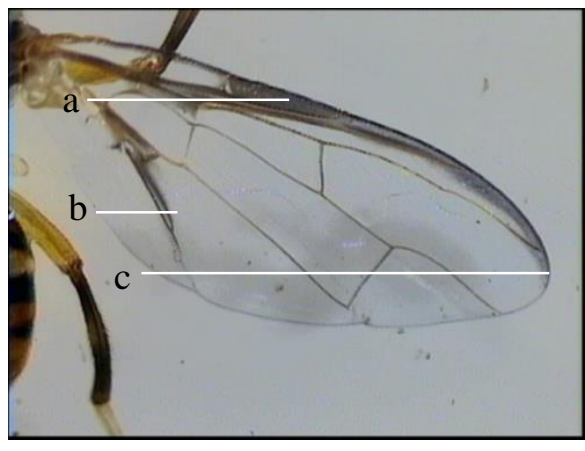

Figure 20 The wing of B. carambolae: (a) costal band, (b) cubital streak, (c) it has no spots

\section{CONCLUSION}

There were five species of fruit fly found in Turikale Subdistrict, Maros District, namely: Bactrocera albistrigata, which attacked Psidium guajava and Syzygium aqueum; B. cucurbitae, which attacked Momordica charantia; B. carambolae which attacked Artocarpus heterophyllust; B. umbrosa which attacked Artocarpus altilis; and B. dorsalis which attacked Mangifera indica, Avverhoa carambola, Capsicum annum, and Solanum melongena.

\section{ACKNOWLEDGMENT}

The authors would like to thank Department of Plant Pests and Diseases, and Department of Biology Hasanuddin University for their supports.

\section{REFERENCES}

[1] S.S. Siwi, H. Purnama, Taxonomy and Bioecology of Important Fruit Flies Bactrocera spp. (Diptera: Tephritidae) In Indonesia. Center for Research and Development of Biotechnology and Agricultural Genetic Resources, Bogor, 2004.

[2] S. Sunarno, The Interest of Insect Pests Fruit Flies Against Various Colored Trap Boards As One Control Technique. J. Agrofor. 6 (2) (2011) 129134

[3] M. Sarjan, Y. Hendro, H. Hery H, Abundance and Composition of Fruit Flies Species on Dry Land in West Lombok District, CropArgo, 2000.

[4] H.A. Kaurow, M. Tulung, J. Pelealu, Identification and Population of Bactrocera spp. in the Chili, Tomato and Siam Pumpkin Crops Eugenia 21(3) (2015) 105-110. DOI: https://doi.org/10.35791/eug.21.3.2015.9692
[5] K. Khaeruddin, P. Hidayat, M. Yayi, Fruit Fly Identification (Diptera: Tephritidae) in Several Regencies in West Sulawesi Province, Bogor Agricultural University, 2015.

[6] S.W. Manwan, Fruit Flies Species that Attack Fruit in Soppeng District, Department of Pests and Plant Diseases, Faculty of Agriculture. Hasanuddin University, 2012.

[7] S. Sulfiani, Fruit Flies Species that Attack Fruit in Wajo District, Department of Pests and Plant Diseases, Faculty of Agriculture, Hasanuddin University, 2012.

[8] F.D. Yuniar, Fruit Fly Identification in Enrekang Regency, Department of Pests and Plant Diseases, Faculty of Agriculture, Hasanuddin University, 2013.

[9] R.A.I. Drew, The Tropical Fruit Flies (Diptera: Tephritidae: Dacinae) of the Australasian \& Oceanian Regions, Memoirs of the Queensland Museum, 1989, 664p.

[10] A. Larasati, Diversity, Distribution and Key Identification of Fruit Flies (Diptera: Tephritidae) in Bogor Regency and Surrounding Areas, Ph.D. Thesis, Bogor Agricultural University, 2012. 